\title{
Influência do Nivel de Emprego Formal na Taxa de Suicidios em Minas Gerais
}

\author{
Laís de Sousa Abreu Soares ${ }^{1}$ (D) | Yan Gabriel Pereira Martins ${ }^{2}$ (D) | Evandro Camargos \\ Teixeira $^{3}$ (iD

\footnotetext{
${ }^{1}$ Mestranda de Economia na Universidade Federal de Viçosa, Viçosa, MG, Brasil. E-mail: laiis.abreu@gmail.com

${ }^{2}$ Bacharel em Economia pela Universidade Federal de Viçosa, Viçosa, MG, Brasil. E-mail: yan.martins@ufv.br

2 Professor Associado ao Departamento de Economia, Universidade Federal de Viçosa, Viçosa, MG, Brasil. E-mail: evandro.teixeira@ufv.br
}

\begin{abstract}
RESUMO
O suicídio é um problema de saúde pública e um fenômeno complexo com expressivas consequências negativas para os indivíduos e para a sociedade. Sua ocorrência é influenciada por diversos fatores, incluindo o mercado de trabalho. Nesse sentido, o presente estudo pretende analisar o efeito do nível de emprego no setor formal sobre as taxas de suicídios dos municípios de Minas Gerais, entre 2002 e 2017. Para tal, foram utilizados dados do Índice Mineiro de Responsabilidade Social, disponibilizados pela Fundação João Pinheiro. Um modelo econométrico com dados em painel dinâmico foi estimado por meio do Método de Momentos Generalizados em Sistema (GMM-SYS). Os resultados mostraram uma influência estatisticamente significante e positiva do nível de emprego formal na taxa de suicídios, ou seja, tal taxa é diminuída. Enfatiza-se, dessa forma, a importância do emprego assistido de direitos para reduzir a instabilidade dos indivíduos e evitar que esses recorram a ações extremas, como o suicídio.
\end{abstract}

\section{PALAVRAS-CHAVE}

Nivel de emprego, Suicídios, Minas Gerais, Painel dinâmico

\section{Influence of the Level of Formal Employment on the Suicide Rate in Minas Gerais}

\begin{abstract}
Suicide is a public health problem and a complex phenomenon with significant negative consequences for individuals and for society. Its occurrence is influenced by several factors, including the labor market. In this sense, the present study aims to analyze the effect of the level of employment in the formal sector on suicide rates in the municipalities of Minas Gerais, between 2002 and 2017. To do so, data from the Mineiro Index of Social Responsibility, made available by the João Pinheiro Foundation, were used. An econometric model with dynamic panel data was estimated using the Generalized System Method of Moments (GMM-SYS). The results showed a statistically significant, positive influence of the level of formal employment on the suicide rate, i.e., such rate is decreased. Thus, the importance of rights-assisted employment is emphasized to reduce the instability of individuals and prevent them from resorting to extreme actions, such as suicide.
\end{abstract}

\section{KEYWORDS}

Employment level, Suicides, Minas Gerais, Dynamic panel

\section{CLASSIFICAÇÃO JEL}

J08, I1, I31 


\section{Introdução}

O suicídio, definido como tentativa de tirar a própria vida que resulta em morte, é reconhecido pela Organização Mundial da Saúde (OMS) como um problema de saúde pública, sendo causa de cerca de 700 mil óbitos mundialmente todos os anos, equivalente a uma morte a cada quarenta segundos. Cada óbito desse tipo afeta famílias, comunidades e países e tem efeitos sociais e econômicos duradouros (World Health Organization, 2019).

O filósofo Durkheim (2000) foi um dos primeiros a estudar o tema ao expor suas ideias na obra "Le Suicide", na qual o autor investiga o que leva um indivíduo a decidir por colocar fim na própria vida. Ele propôs que os suicídios se enquadram em três categorias: suicídio egoísta, no qual o indivíduo o faz porque não sente mais a conexão entre si e a sociedade; suicídio altruísta, aquele cometido seguindo comportamentos do grupo do qual faz parte ou com a intenção de gerar um bem coletivo; e suicídio anômico, cometido após mudanças drásticas na sociedade, como perdas de poder, mudanças de status social ou empobrecimento.

É possível perceber, pela definição e pelas diferentes classificações, que o suicídio é um fenômeno complexo que tem relação com fatores individuais e sociais. Por esse motivo, é objeto de estudo de uma gama variada de Ciências, incluindo Biologia, Psicologia, Medicina, Sociologia e Economia. Especificamente, a análise econômica do tema, além de se dedicar às consequências desse fenômeno na economia, evidencia o estudo da interação entre fatores socioeconômicos e taxas de suicídio, ou seja, analisa se existe influência de determinados fatores na sua ocorrência.

Em relação às consequências econômicas dos suicídios, a literatura destaca a necessidade de aumento do dispêndio do Estado com saúde pública; a ausência no mercado de trabalho daqueles indivíduos que tentam tirar a própria vida e a redução na mão de obra quando as tentativas resultam em óbito; a diminuição do nível de bem-estar das pessoas próximas, particularmente dos familiares, que possivelmente apresentarão redução da sua produtividade, dentre outras (Godoi, 2018). Nesse sentido, as consequências produtivas dos suicídios, segundo a World Health Organization (2017), fizeram com que mais de 54 milhões de anos acumulados tenham sido vividos com incapacidade (Years Lived with Disability - YLD), demonstrando a magnitude do prejuízo para a economia.

Na direção oposta, ou seja, no tocante ao impacto de fatores socioeconômicos na ocorrência de suicídios, a literatura destaca aspectos culturais, demográficos, econômicos e sociais. Um exemplo é o estudo de Bertolote e Alexandra (2002), que verificou que países com predominância de religiões mais propensas a tratar o autoextermínio com maior rejeição tendem a apresentar taxas de suicídio menores em relação àqueles onde prevalecem religiões com menor rejeição ou compostos em sua maioria por indivíduos ateus. Já os estudos de Machado e Santos (2015) e Nock et al. (2008) encontram diferenças significativas quando comparadas as taxas de suicídios entre 
os sexos.

A influência de fatores como renda, desigualdade e desemprego nas taxas de suicídio também é objeto de estudo da literatura concernente ao tema. Durkheim (2000) já apontava que grande parte do estresse social que acomete os indivíduos é causado por rápidas mudanças culturais e por flutuações econômicas drásticas, aumentando a ocorrência de suicídios. Em períodos de crises econômicas, como no caso das crises de 1929 e de 2008, por exemplo, costuma-se registrar um aumento significativo nas taxas de suicídios (Tapia Granados e Diez Roux, 2009; Stuckler et al., 2011). Dessa forma, uma situação econômica mais estável, tanto a nível macro quanto microeconômico, permite que os indivíduos tenham acesso de forma mais adequada às suas necessidades e, consequentemente, passem por menos situações as quais podem levar a comportamentos extremos, como é o caso do suicídio.

De acordo com Costa e Ludemir (2005), tem sido verificada a associação entre transtornos mentais (que levam à ocorrência de suicídios) com eventos vitais produtores de estresse, como baixo apoio social e variáveis relativas às condições de vida e trabalho como baixa escolaridade, menor posse de bens duráveis, condições precárias de moradia, desemprego e informalidade nas relações de trabalho. Nesse sentido, Kposowa (2001) aponta que o emprego, além de ser a fonte de renda mais comum para a população em geral, permitindo que necessidades básicas sejam atendidas, é uma forma de integração das pessoas à sociedade. De acordo com o autor, a perda desse enfraquece a integração social do indivíduo, o privando do seu status e do seu papel social, e aumenta o isolamento, amplificando o risco de doenças mentais, como ansiedade e depressão, e a ocorrência de suicídio.

No que tange à literatura, é possivel encontrar na literatura internacional um maior número de trabalhos desenvolvidos que analisaram os fatores socioeconômicos e demográficos que influenciam as taxas de suicídios. Por outro lado, na literatura nacional, tais trabalhos são mais raros, geralmente voltados para a área da saúde. Mas, isso não significa que a quantidade de suicídios nacionais não é significativa. De acordo com a World Health Organization (2014), o Brasil é o oitavo país com mais suicídios no mundo, atrás de Índia, China, Estados Unidos, Rússia, Japão, Coréia do Sul e Paquistão. Em 2013, o país contabilizou mais de 11 mil óbitos causados por suicídio. Além disso, considerando que as ocorrências de suicídios estão ligadas ao acometimento por transtorno depressivo (World Health Organization, 2002), o Brasil encontra-se em uma posição preocupante, já que 5,8\% de sua população foi diagnosticada com a doença, posicionando o país acima da média global de 4,4\% e em primeiro lugar na América Latina ${ }^{1}$.

Dentro dos limites territoriais do Brasil, um país continental, há grandes diferenças socioculturais entre as regiões, o que implica também fortes discrepâncias com relação às taxas de suicídios. Apesar de Godoi (2018) destacar que a região Sudeste

\footnotetext{
${ }^{1}$ Dados da cartilha da OMS intitulada “Depression and Other Common Mental Disorders”, publicada em 2017.
} 
apresentou menor aumento relativo na referida taxa nos últimos anos, Minas Gerais, especificamente, merece destaque, pois o número absoluto de suicídios foi o segundo mais elevado do país em $2017^{2}$, ficando atrás somente do estado de São Paulo. Além disso, a taxa de mortalidade por autoextermínio no mesmo ano foi de 7,17/100 mil habitantes, a nona maior entre todas as Unidades Federativas do país e superior à taxa nacional, que foi igual a 6,02/100 mil habitantes ${ }^{3}$.

Diante dos números apresentados, da lacuna literária e da importância de se reduzir as taxas de suicídios em razão das suas consequências negativas sobre a sociedade, o presente estudo pretende analisar o efeito do nível de emprego formal nas taxas de suicídios no estado de Minas Gerais entre 2002 e 2017, utilizando dados do Índice Mineiro de Responsabilidade Social (IMRS), desenvolvido pela Fundação João Pinheiro (FJP).

Assim, pretende-se contribuir para a literatura ao determinar o impacto de uma variável que representa a estabilidade econômica do indivíduo (taxa de emprego formal) na ocorrência de suicídios, utilizando um modelo econométrico, sendo que a análise versará sobre Minas Gerais, um estado economicamente heterogêneo e que possui números expressivos em relação aos casos de suicídios. Ou seja, avalia-se não apenas a influência da capacidade do indivíduo em atender suas necessidades, através do emprego, mas também do nível de estabilidade e dos direitos que esse emprego representa, através da formalização. Mediante os resultados encontrados, espera-se que seja possivel a implementação de políticas públicas no intuito de aumentar a quantidade de postos de trabalho formais no estado e apoiar os indivíduos com problemas relacionados à saúde mental, para que, consequentemente, haja mitigação das taxas de suicídios.

Além da presente introdução, o artigo está dividido em mais quatro seções. Na próxima seção, são apresentados aspectos teóricos e empíricos do estudo econômico relacionado ao tema, e, em seguida, encontra-se a metodologia. Então, os resultados são discutidos, com posterior apresentação das considerações finais.

\section{Aspectos Teóricos e Empíricos}

Um dos primeiros estudos relacionados ao suicídio no âmbito econômico foi o de Hamermesh e Soss (1974), no qual os autores desenvolveram um modelo teórico, baseado na microeconomia neoclássica (utilizando o conceito de utilidade). Por meio desse modelo, realizaram uma análise empírica dos determinantes dos suicídios em países desenvolvidos no período de 1947-67. Os autores reconheceram a incapacidade da análise estatística em abranger todos os aspectos do fenômeno, mas demonstraram o valor de se utilizar a teoria econômica para analisá-lo. Os resultados mostraram, assim como estudos de outras áreas, relação entre suicídios e idade, renda e ocupação.

\footnotetext{
${ }^{2}$ Dados oriundos do DataSUS: mortes por causas externas; categorias da CID-10 entre X60-X84.

${ }^{3}$ Cálculo com base nos dados da soma do número de mortos por causas externas entre as categorias X60-X84 da CID-10 e a estimativa populacional do IBGE para o ano de 2017.
} 
A principal conclusão foi de que o nível de renda influencia negativamente o número de suicídios, com efeito marginal cada vez menor à medida que ocorrem acréscimos em seu nível.

Após o estudo de Hamermesh e Soss (1974) demonstrar a possibilidade e a importância de se estudarem os suicídios pela ótica econômica, a literatura empírica sobre o tema cresceu significativamente, inclusive no tocante à relação específica entre desemprego e suicídios. O trabalho de Morrell et al. (1993), por exemplo, realizou uma análise agregada (ecológica) entre taxas de suicídios anuais de homens e mulheres e taxas de desemprego na Austrália, entre 1907 e 1990, utilizando métodos que levam em consideração a autocorrelação de dados em séries temporais. Os resultados confirmaram a associação positiva entre as variáveis no caso dos homens, de forma que essa associação foi mais intensa para indivíduos entre 15 e 24 anos.

De forma semelhante, o estudo de Iglesias-Garcia et al. (2017) pretendeu determinar a associação entre desemprego e suicídio e investigar se essa associação é afetada por mudanças no ciclo econômico ou outras variáveis, como idade e sexo. Através da análise de tendência temporal, foi analisada a mudança no número de suicídios entre 1999 e 2013 na Espanha. Para tal, foram utilizadas correlações de Pearson e modelos de regressão, que indicaram uma significativa associação positiva entre desemprego e suicídio entre homens. No período, $1 \%$ do aumento anual do desemprego foi relacionado a $6,9 \%$ de aumento na taxa de suicídios da população total e 9,04\% na taxa de suicídios de homens economicamente ativos.

A relação entre desemprego e suicídios é, portanto, parte extensa da literatura econômica sobre o tema. Análises que se aproximam do objetivo principal do presente estudo, de determinar a influência do emprego formal sobre taxas de suicídios, são mais raras. Um exemplo é o estudo empírico realizado por Kim e Cho (2017), que utilizaram informações de vinte países participantes da Organização para Cooperação e Desenvolvimento Econômico (OCDE) durante 1994 e 2010 e por meio de dados em painel com efeitos aleatórios analisaram a relação entre a estabilidade do emprego e os suicídios entre pessoas de 20 a 34 anos. Os autores concluíram que um baixo nível de proteção trabalhista foi associado a um aumento nas taxas de suicídio em todas as populações estudadas.

Entre os estudos mais recentes, destacam-se aquele realizado por Kaufman et al. (2020), o qual indicou que aumentos no salário mínimo podem reduzir as taxas de suicídios nos estados americanos, principalmente quando os níveis de desemprego são altos; o estudo de Chang e Chen (2017), no qual os resultados sugeriram que taxas de suicídios foram pró-cíclicas em relação ao ciclo de negócios (medido pela taxa de desemprego) no Estados Unidos, entre 1928 e 2013; e o trabalho de Basta et al. (2018), que analisaram o efeito da crise econômica da Grécia na taxa de suicídios na região de Creta.

Além da possibilidade de realização de estudos com dados agregados, como os su- 
pracitados, existem estudos que analisam a relação entre emprego ou desemprego e suicídios a partir de dados individuais. Nesse contexto, Blakely et al. (2003) pretenderam determinar a associação entre status da força de trabalho e posição socioeconômica na Nova Zelândia e ocorrência de suicídios. Utilizando dados de 2,04 milhões de participantes do CENSO de 1991 do país e estimando um modelo de regressão logística, os autores encontraram resultados os quais indicaram que homens e mulheres sem emprego têm 2,35 e 2,42 vezes mais chances, respectivamente, de cometerem suicídios em relação aos que estão empregados.

Mais recentemente, entre os estudos que investigaram a relação entre mercado de trabalho e suicídios, utilizando dados individuais, destaca-se o estudo de Ludin et al. (2012), a partir da população da cidade de Estocolmo; e o trabalho de Milner et al. (2012), que utilizou dados da população australiana para investigar o efeito do desemprego de longo prazo na probabilidade de ocorrência de suicídio, entre outros.

No Brasil, dentre os poucos trabalhos realizados referentes à temática no âmbito da Economia, destaca-se o de Loureiro et al. (2013). Os autores utilizaram informações dos vinte e seis estados brasileiros mais o Distrito Federal durante o período 19802009 por meio de três modelos de regressão, um por Mínimos Quadrados Ordinários (MQO), outro por Efeitos Fixos (EF) e o último por Diferenças em Diferenças (DPD). Loureiro et al. (2013) concluíram que os efeitos das taxas de desemprego sobre as taxas de suicídios se evidenciam principalmente entre as pessoas jovens, com idade entre 15 e 29 anos, predominantemente do sexo masculino. Os autores ainda constataram que as elasticidades taxa de suicídios - taxa de desemprego, estimadas através dos modelos de EF e DPD, foram de 5,95 e 3,88, respectivamente.

Com o objetivo de estimar a relação entre o cenário socioeconômico e o número de suicídios nos municípios brasileiros, com inserção da variável de desemprego e tendo como foco os municípios do Rio Grande do Sul, Fraga et al. (2017) utilizaram um modelo de Regressão Quantílica. Os autores encontraram relação inversa e significativa entre a taxa de desemprego e o número de suicídios. Para explicar esse resultado, os autores se apoiaram em Gerdtham e Ruhm (2006), destacando que indivíduos empregados em jornadas intensas de trabalho e com muito esforço físico podem desenvolver um quadro de estresse prejudicial para a saúde física e mental. Outra explicação evidenciada pelos autores para a relação inversa encontrada no Brasil e no estado do Rio Grande do Sul foi a existência de programas de seguro-desemprego, que podem mitigar os efeitos psicológicos do desemprego.

De modo geral, percebe-se que uma considerável parte dos estudos que analisa a relação entre suicídios e mercado de trabalho tem como foco a influência do desemprego e sua duração na ocorrência do fenômeno, sendo significativamente menor o número de análises relacionadas ao nível de emprego e suas especificidades, incluindo o fato de ser formal ou informal. Desse modo, tendo em vista a lacuna da literatura, o presente estudo pretende determinar o efeito do emprego formal nas taxas de suicídios no estado de Minas Gerais, considerando os dados dos municípios 
no período de 2002 a 2017. Portanto, por meio de um estudo ecológico (com dados agregados), há expectativa de que em Minas Gerais a variável taxa de emprego no setor formal impacte negativamente a taxa de suicídios e que esse efeito seja estatisticamente significativo.

\section{Metodologia}

Para o desenvolvimento do trabalho, optou-se pela utilização de um painel de dados dinâmico, que permite mensurar o impacto da taxa de emprego no setor formal sobre a taxa de suicídios. Este painel foi constituído a partir de dados dos 853 municípios mineiros (unidades de observação) durante o período de 2002 a 2017. O período analisado mantém a consistência de unidades de observação, visto que desde o ano de 2000 o estado mantém os mesmos 853 municipios. A disponibilidade das informações foi fator que limitou a análise entre 2002 e 2017. A fonte principal dos dados utilizados para a construção das variáveis é o Índice Mineiro de Responsabilidade Social (IMRS), desenvolvido pela Fundação João Pinheiro (FJP). Nele, encontram-se diversas informações socioeconômicas referentes ao estado de Minas Gerais e seus municípios.

De acordo com Santos e Kassouf (2008), a estrutura de dados em painel é apropriada, pois permite que sejam exploradas as dimensões espacial e temporal, que é o caso desta pesquisa. Além disso, a escolha de se utilizar painel de dados de forma dinâmica permite que sejam controladas as possiveis relações de endogeneidade entre algumas variáveis, além de considerar a existência de uma relação dinâmica ao incluir a variável explicada defasada em 1 lag.

O estimador utilizado é o de Método de Momentos Generalizados em Sistema GMM-SYS, proposto por Blundell e Bond (1998), apoiando-se no trabalho de Arellano e Bover (1995). Desse modo, o modelo a ser estimado é o seguinte:

$$
\begin{aligned}
& L N T X S U I C_{i, t}=\alpha+\beta_{1} L N T X S U I C_{i, t-1}+\beta_{2} \text { TXEMPFORM }_{i, t} \\
& +\beta_{3} S A U ́ D E_{i t}+\beta_{4} A S S S O C I A L_{i, t} \\
& +\beta_{5} E D U C A C ̧ \tilde{A} O_{i, t}+\beta_{6} \text { PIBPERCAPIT } A_{i, t} \\
& +\beta_{7} D E N S D E M O G R_{i, t} \\
& +\beta_{8} E V E L H E C I M E N T O_{i, t} \\
& +\beta_{9} \text { TXMORTAGRESS } S_{i, t}+\varepsilon_{i, t}
\end{aligned}
$$

Sendo LNTXSUIC $C_{i, t}$ o logaritmo das taxas de suicídios dos municípios mineiros por 100 mil habitantes, que corresponde à variável dependente utilizada como proxy para quantificar os suicídios, $\alpha$ é o intercepto, os $\beta$ 's são os parâmetros a serem estimados e $\varepsilon$ é o erro aleatório. Portanto, a forma funcional utilizada é log-linear. 
É importante salientar que a escolha das variáveis explicativas inseridas no modelo teve como critério de seleção a literatura referente ao tema. As referidas variáveis, assim como suas descrições e sinais esperados, são apresentadas na Tabela 1.

Tabela 1. Descrição das variáveis a serem utilizadas na estimação do modelo econométrico

\begin{tabular}{|c|c|c|}
\hline Variável & Descrição & Sinal esperado \\
\hline$L N T X S U I C_{i, t}$ & $\begin{array}{l}\text { Logaritmo da taxa de suicídios (número de } \\
\text { mortes por suicídio no município para cada } \\
100 \text { mil habitantes) }\end{array}$ & $\begin{array}{c}\text { Variável } \\
\text { dependente }\end{array}$ \\
\hline$L N T X S U I C_{i, t-1}$ & $\begin{array}{l}\text { Logaritmo da taxa de suicídios defasada em } 1 \\
\text { período }\end{array}$ & Positivo \\
\hline$T X E M P F O R M_{i, t}$ & $\begin{array}{l}\text { Número de empregos formais do município di- } \\
\text { vidido pela população (entre } 16 \text { e } 64 \text { anos) } \\
\text { ativa residente, multiplicado por } 100\end{array}$ & Negativo \\
\hline$S A U ́ D E_{i t}$ & Gastos per capita com saúde & Negativo \\
\hline$A S S S O C I A L_{i, t}$ & Gastos per capita com assistência social & Negativo \\
\hline$E D U C A C ̧ \tilde{A} O_{i, t}$ & Gastos per capita com educação & Negativo \\
\hline PIBPERCAPIT $A_{i, t}$ & PIB per capita & Negativo \\
\hline$D E N S D E M O G R_{i, t}$ & Densidade demográfica & (Relação ambígua) \\
\hline$E V E L H E C I M E N T O_{i, t}$ & $\begin{array}{l}\text { Taxa de envelhecimento populacional: nú- } \\
\text { mero de pessoas residentes de } 65 \text { anos ou } \\
\text { mais dividido pela população, multiplicado } \\
\text { por } 100 \text {. }\end{array}$ & Positivo \\
\hline$T X M O R T A G R E S S_{i, t}$ & $\begin{array}{l}\text { Razão entre o número absoluto de vítimas fa- } \\
\text { tais de agressão e a população do municipio, } \\
\text { multiplicado por } 100 \text { mil }\end{array}$ & Positivo \\
\hline
\end{tabular}

Fonte: Elaboração própria.

Utiliza-se o logaritmo da taxa de suicídios com defasagem de um período como uma das variáveis explicativas, $L N T X S U I C_{i, t-1}$, que é um pressuposto dos modelos dinâmicos. Essa variável defasada corresponde, portanto, ao "efeito inércia", no qual as taxas de suicídios do ano anterior explicam, em parte, as taxas de suicídios do ano seguinte. Nesse caso, é possivel que as taxas de suicídios do período anterior funcionem como um mecanismo de encorajamento ou incentivo para indivíduos que sofrem com problemas de saúde mental. Portanto, o sinal esperado para essa variável é positivo.

A Taxa de Emprego no Setor Formal, TXEMPFORM ${ }_{i, t}$, corresponde à variável central do presente estudo. Espera-se que essa variável tenha sinal negativo. Também foram inseridas no modelo as variáveis Gasto Público per capita com Saúde e Gasto Público per capita com Assistência Social. Optou-se pela utilização dessas variáveis, 
pois, ao fornecer mais recursos para essas áreas, espera-se que os indivíduos, no geral, recebam um suporte mais adequado para sua saúde mental, reduzindo, assim, as taxas de suicídios. Além disso, mesmo que realizem a tentativa de suicídio, talvez não cheguem ao óbito devido a uma atuação mais eficiente dos órgãos ligados ao setor de saúde. Concomitantemente, essas variáveis são também influenciadas pelas taxas de suicídios à medida que o governo, possivelmente, baseia os Gastos per capita com Saúde e Assistência Social, em parte, a partir das referidas taxas, denotando, assim, uma relação de endogeneidade, atestada por meio do teste de Hausman.

O controle dessa relação endógena entre as variáveis se dá através do método utilizado neste trabalho, no qual são utilizadas as próprias variáveis defasadas em dois períodos como instrumentos. Segundo Vieira et al. (2016), diversas variáveis se relacionam entre si e com seus valores passados. Logo, quando se utiliza a defasagem da variável explicada como variável explicativa, é possível a obtenção de estimadores não viesados. Do contrário, caso fosse utilizado um painel de dados estático, as possíveis relações endógenas entre algumas variáveis não seriam controladas. Além disso, a relação endógena presente no modelo também foi controlada através da inserção de dois instrumentos adicionais, que foram o Gasto Público per capita com Infraestrutura e o Gasto Público per capita com Habitação. É importante salientar que as variáveis de gastos foram deflacionadas para o ano de 2017 através do Índice Nacional de Preços ao Consumidor (INPC), divulgado pelo Índice Brasileiro de Geografia e Estatística (IBGE).

A variável Gasto Público per capita com Educação, $E D U C A C O_{i, t}$, também foi inserida no modelo, pois essa é uma das formas de se realizar a prevenção dos suicídios. Segundo Blakely et al. (2003), o baixo nível educacional está associado ao risco mais elevado de suicídios. Portanto, espera-se que o aumento nos gastos per capita com educação reduza as taxas de suicídios, indicando dessa forma um sinal negativo esperado para a variável.

Como proxy das condições econômicas gerais dos municípios, optou-se pela utilização da variável PIB per capita, PIBPERCAPIT $A_{i, t}$, tendo em vista que essa pode ser um bom indicativo de recessão econômica e pressão sobre o nível de emprego. Logo, espera-se que essa variável tenha sinal negativo ao impactar as taxas de suicídios, já que a queda no nivel de renda pode se tornar um fardo, principalmente para os homens, que sofrem pressão pelo "male breadwinner system"4, resultando em aumento nos suicídios (Kim e Cho, 2017; Reeves et al., 2015).

Também foi utilizada como variável de controle no modelo a Densidade Demográfica dos municípios mineiros, $D E N S D E M O G R_{i, t}$. A intenção é que essa variável demonstre se há diferença entre residir em um local mais ou menos populoso, visto que há considerável ambiguidade nos trabalhos que utilizaram a densidade populacional como variável explicativa. Segundo Loureiro et al. (2015), em um estudo para

\footnotetext{
${ }^{4}$ Teoria que denota que os homens sofrem pressão por exercerem o papel de provedores das famílias.
} 
Portugal, elevadas taxas de internação por distúrbios psicológicos estão associadas a grandes densidades populacionais. Entretanto, segundo Gonçalves e Lourival (2011) e Helbich et al. (2017), áreas não urbanizadas estão ligadas ao elevado risco de suicídios em razão do isolamento, o que demonstra divergência em relação ao sinal esperado para a variável. De acordo com Isometsä et al. (1997), a questão é complexa, pois existe diferença entre os suicídios cometidos em áreas urbana e rural, sendo aqueles ocorridos no meio urbano motivados, em sua maioria, por distúrbios psicológicos; e os suicídios rurais motivados por experiências relacionadas à saúde física. Dito isso, é difícil apontar se a relação entre as taxas de suicídios e a densidade populacional é negativa ou positiva.

Outra variável utilizada é o Índice de Envelhecimento, ENVELHECI$M E N T O_{i, t}$. Segundo Machado e Santos (2015), as maiores taxas de suicídios são verificadas entre os idosos. Portanto, espera-se que, quanto maior for a proporção de população idosa em determinada localidade, mais elevada será a taxa de suicídios dessa.

Como as mortes por agressão podem causar um impacto psicológico nos indivíduos, a referida variável foi inserida no modelo, TXMORTAGRES $S_{i, t}$. Acredita-se que, quanto maior essa taxa, mais elevadas as chances de que as pessoas se sintam inseguras, o que pode aumentar as taxas de suicídios. Uma compreensão semelhante foi apresentada na pesquisa de Gonçalves e Lourival (2011) através da variável taxa de criminalidade, a qual tinha relação positiva com a taxa de suicídios. Além disso, quando um indivíduo perde um ente querido por agressão, os efeitos psicológicos podem ser severos e resultar até mesmo em autoextermínio. Portanto, espera-se que a variável tenha sinal positivo. Em sequência, são realizados os testes de Sargan e de correlação serial de Arellano-Bond, tipicamente utilizados em dados de painel dinâmico. O objetivo do primeiro teste é analisar a validade conjunta dos instrumentos utilizados, ou seja, se estes são independentes do termo de erro. Com relação ao segundo teste, o objetivo é verificar a correlação serial dos erros.

\section{Resultados}

Esta seção tem como objetivo a apresentação dos resultados da pesquisa e está dividida em duas subseções. Na primeira, é realizada a análise descritiva e na segunda são apresentados os resultados encontrados após a estimação do modelo econométrico.

\subsection{Análise Descritiva}

Convém, inicialmente, apresentar as médias e os desvios-padrão das variáveis inseridas no modelo econométrico a ser estimado, vide Tabela 2, abaixo. Como é possível perceber, os desvios-padrão, em geral, são elevados, o que evidência a heterogeneidade entre os municípios mineiros. A variável Taxa de Suicídios é um bom exemplo, haja 
vista que o desvio-padrão é maior que a própria média. Os dados de Máximo e Mínimo também demonstram a referida heterogeneidade de forma que alguns municípios não tiveram mortes por autoextermínio em 2017, enquanto São Sebastião do Rio Preto, por exemplo, apresentou uma taxa de 127,71 mortos por 100 mil habitantes.

Tabela 2. Estatísticas descritivas

\begin{tabular}{lcccc}
\hline \hline & Média & $\begin{array}{c}\text { Desvio } \\
\text { Padrão }\end{array}$ & Máximo & Mínimo \\
\hline Taxa de Suicídios por 100 mil habitantes & 8,99 & 13,77 & 127,71 & 0 \\
Taxa de emprego no setor formal & 21,5 & 12,39 & 130,30 & 4,70 \\
Gastos per capita com saúde & 693,53 & 300,57 & 3766,40 & 193,99 \\
Gastos per capita com assistência social & 81,88 & 67,53 & 1080,97 & 0 \\
Gastos per capita com educação & 645,59 & 260,81 & 3659,33 & 212,91 \\
PIB per capita & 18817,8 & 19437,2 & 289925,4 & 6087,8 \\
Densidade demográfica & 70,47 & 335,13 & 7566,24 & 1,35 \\
Envelhecimento & 43,07 & 4,17 & 55,85 & 29,60 \\
\hline \hline
\end{tabular}

Fonte: Elaboração própria.

A variável Taxa de Emprego no Setor Formal também apresenta elevada heterogeneidade, apesar de possuir desvio-padrão consideravelmente abaixo da média. No caso dessa variável, a análise de Máximo e Mínimo também demonstra as grandes diferenças entre os municípios, de modo que o município com maior percentual de emprego no setor formal dividido pela população ativa (entre 16 e 64 anos), Comendador Gomes, apresentou uma taxa de $130,30^{5}$, contra os 4,7 da cidade de Simonésia.

O mesmo comportamento heterogêneo é verificado com as variáveis PIB per capita e Densidade demográfica, as quais possuem desvio-padrão maior que a média e grande disparidade entre o Mínimo e o Máximo. A respeito das variáveis relativas aos gastos públicos per capita, percebe-se que, apesar de os desvios-padrão não serem muito elevados, a quantidade de recursos financeiros per capita disponibilizada pelos governos municipais é, de certo modo, discrepante entre uma cidade e outra. Isso pode ser observado nas colunas de Máximo e Mínimo. Nessas, nota-se que os municípios com os menores gastos per capita dispendem menos de $6 \%$ em relação àqueles que mais gastam, demonstrando como as mesorregiões mineiras são heterogêneas, até mesmo em relação às políticas públicas, haja vista as grandes diferenças nos níveis de dependência dos municípios em relação aos repasses do Fundo de Participação Municipal (FPM) (Massardi e Abrantes, 2016).

\footnotetext{
${ }^{5} \mathrm{O}$ número percentual de emprego formal em relação à população economicamente ativa é maior que 100 quando o município possui mais cargos de empregos formais que residentes em idade ativa, ou seja, parte da mão de obra formal não reside no município.
} 
Especificamente em relação ao Gasto per capita com Assistência Social, esse chega a ser zero em alguns dos municípios. Isso porque as subfunções consideradas nesse tipo de gasto não fazem parte das despesas obrigatórias (assistência ao idoso; assistência ao portador de deficiência; assistência à criança e ao adolescente; assistência comunitária, custódia e reintegração social; direitos individuais coletivos e difusos e assistência aos povos indígenas).

Em uma perspectiva distinta está a variável de Envelhecimento. Esse índice denota homogeneidade para os municípios mineiros e mostra como o processo de envelhecimento segue evoluindo de forma semelhante entre as cidades, seguindo a tendência mundial e nacional de transição demográfica.

Como o objetivo principal do trabalho é analisar a relação entre as Taxas de Suicídios conjuntamente com as Taxas de Emprego no Setor Formal, as Figuras 1 e 2, a seguir, apresentam respectivamente, a distribuição espacial das taxas de suicídio e das taxas de emprego formal tanto em 2002 quanto em 2017. É possivel verificar, dessa forma, a evolução das taxas de suicídios e de emprego formal nos municípios de Minas Gerais, bem como a relação espacial entre as duas taxas nos dois anos considerados. Considerando as taxas de suicídio em 2002 e 2017 (Figura 1), verifica-se que o número de municípios com Taxa de Suicídios acima de 10/100 mil habitantes aumentou consideravelmente e de forma generalizada entre o primeiro e o último. É importante mencionar que essa elevação no período acompanha o movimento ascendente da Taxa de Suicídios no continente americano, contrariamente ao que ocorre em outros continentes, como demonstrado pela World Health Organization (2019). Além disso, cabe ainda salientar que as taxas acima desse patamar estão muito acima da taxa nacional de 6,02/100 mil habitantes e daquela verificada para o estado de Minas Gerais como um todo, correspondente a 7,17/100 mil habitantes.

Por outro lado, considerando-se as taxas de emprego formal (Figura 2), nota-se que o número de municípios com Taxa de Emprego no Setor Formal acima de 30\% aumentou principalmente nas regiões Sul e Sudoeste do estado mineiro. Apesar do aumento médio total da taxa de suicídios ser considerável em todo estado, também é possivel perceber que as maiores taxas, tanto em 2002 quanto 2017, concentravamse também nas regiões Sul e Sudoeste, assim como no caso do emprego formal. Tal constatação contraria as expectativas de uma associação negativa entre o emprego formal e os suicídios. É importante destacar, no entanto, que a análise descrita é apenas um estudo preliminar dos dados.

Com o intuito de facilitar a visualização dessas variáveis para cada mesorregião mineira, foi elaborada a Tabela 3, a seguir, tendo como base o período 2002-2017. Desse modo, verifica-se que houve aumento médio das taxas de emprego e suicídios em todas as mesorregiões. Pela observação da Tabela 3, destacam-se as estatísticas da mesorregião Oeste de Minas, a qual, em 2002, apresentava a maior taxa média de suicídios do estado e, concomitantemente, a segunda maior taxa de emprego formal. Já a mesorregião do Vale do Mucuri, que em 2002 apresentava a menor taxa média de 
suicídios do estado, mostrava-se uma das regiões com menor taxa de emprego formal. Dessa forma, assim como no caso das Figuras 1 e 2, tais estatísticas contrariam a perspectiva de associação negativa entre as variáveis supracitadas.

Figura 1. Distribuição espacial das taxas de suicídios em 2002 e 2017

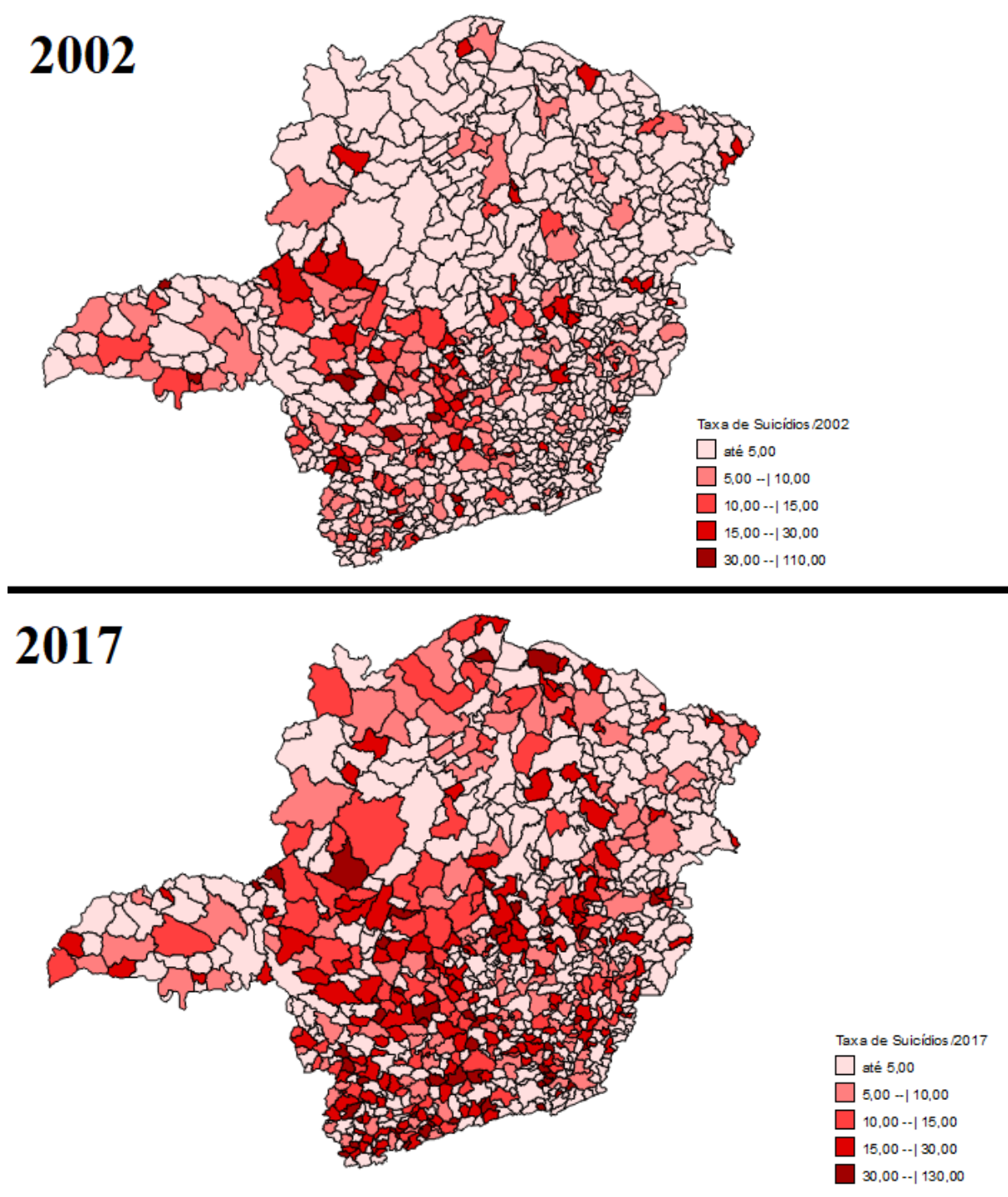

Fonte: Elaboração própria.

O caso do Vale do Mucuri pode causar certa estranheza pelo aumento desproporcional de 5657\% na média da Taxa de Suicídios, entretanto, há de se ressaltar que em 2002 essa taxa era extremamente baixa. Logo, mesmo que essa variável tenha apresentado um valor razoavelmente pequeno em 2017, o aumento percentual calculado entre o primeiro e o último ano da amostra acabou sendo muito elevado. Em 2017, o Vale do Mucuri se mantém como mesorregião com menor taxa média de suicídios, 
Figura 2. Distribuição espacial das taxas de emprego formal em 2002 e 2017
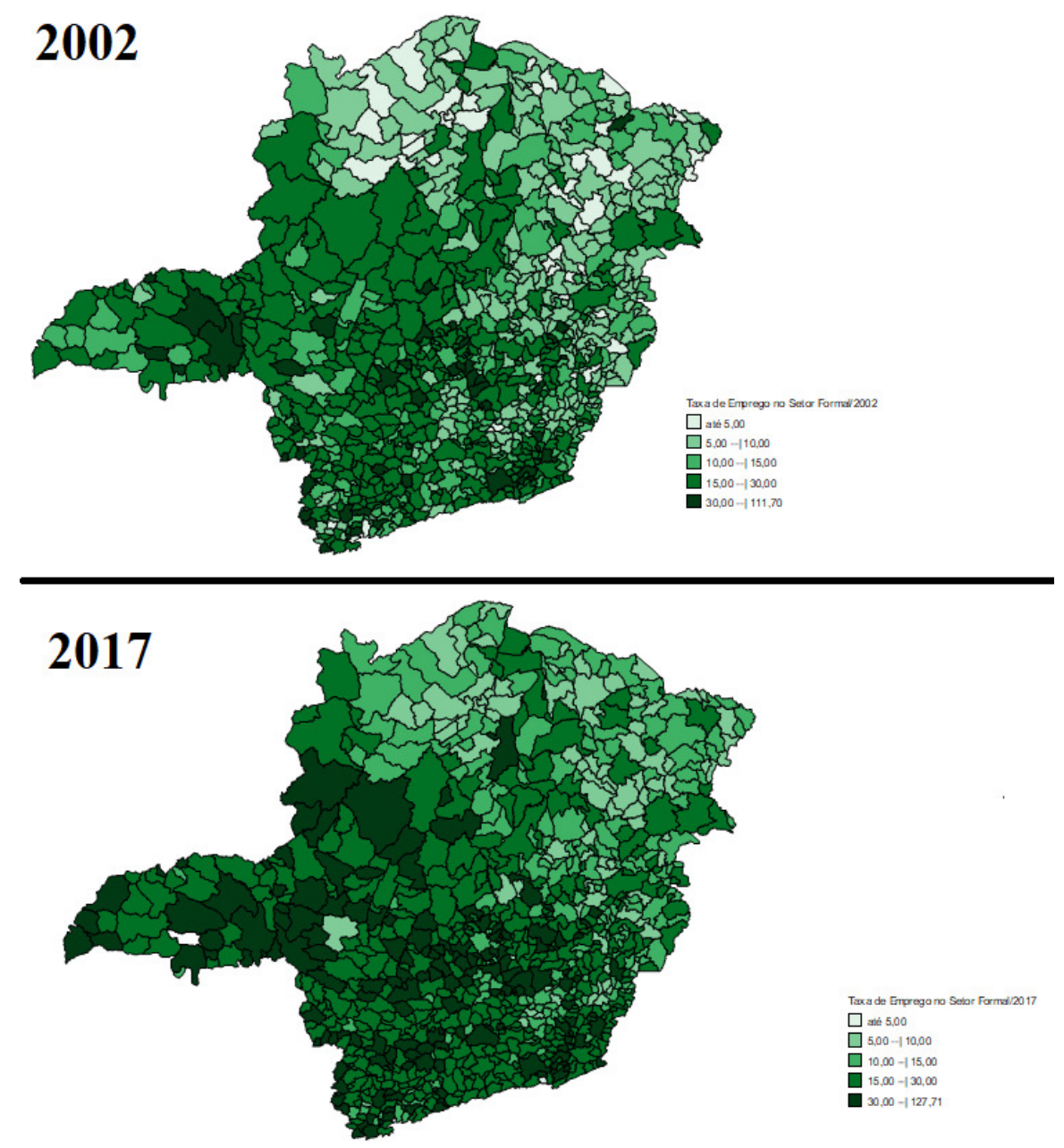

Fonte: Elaboração própria.

apesar do crescimento observado, e se tornou a região com menor taxa de emprego formal do estado.

A análise descritiva realizada, portanto, aponta para uma relação positiva entre a taxa de empregos formal e a taxa de suicídios. No entanto, é importante destacar que essa desconsidera aspectos importantes, como a influência de diversas outras variáveis socioeconômicas na ocorrência do fenômeno. Dessa forma, a subseção a seguir traz os resultados da análise econométrica realizada.

\subsection{Resultados Econométricos}

Nesta subseção, são apresentados os resultados econométricos, vide Tabela 4, a seguir. Foram estimados quatro modelos: um modelo de Mínimos Quadrados Ordinários - Pooled para dados em painel, um modelo de efeitos fixos, um modelo com estimador GMM-Difference e, por fim, um modelo com estimador GMM-System. Tal procedimento foi realizado para demonstrar a robustez dos resultados e avaliar qual 
Tabela 3. Médias das Taxas de Emprego no Setor Formal e das Taxas de Suicídios por mesorregião de Minas Gerais em 2002 e 2017

\begin{tabular}{lcccccc}
\hline \hline & \multicolumn{2}{c}{$\begin{array}{c}\text { Média da Taxa de Emprego } \\
\text { no Setor Formal }\end{array}$} & \multicolumn{2}{c}{ Média da Taxa de Suicídios } \\
\hline Mesorregiões & 2002 & 2017 & $\begin{array}{c}\text { Aumento } \\
\%\end{array}$ & 2002 & 2017 & $\begin{array}{c}\text { Aumento } \\
\%\end{array}$ \\
\hline Metropolitana de Belo Horizonte & 18,19 & 25,81 & $42 \%$ & 4,64 & 8,80 & $\mathbf{8 9 \%}$ \\
Campo das Vertentes & 15,21 & 22,94 & $51 \%$ & 5,53 & 13,81 & $\mathbf{1 5 0 \%}$ \\
Central Mineira & 19,42 & 24,25 & $25 \%$ & 4,51 & 15,26 & $\mathbf{2 3 9 \%}$ \\
Jequitinhonha & 9,43 & 12,66 & $34 \%$ & 2,13 & 5,11 & $\mathbf{1 4 0 \%}$ \\
Noroeste de Minas & 14,74 & 23,89 & $62 \%$ & 5,05 & 7,48 & $\mathbf{4 8 \%}$ \\
Norte de Minas & 8,77 & 13,74 & $57 \%$ & 1,80 & 6,08 & $\mathbf{2 3 8 \%}$ \\
Oeste de Minas & 20,17 & 30,37 & $51 \%$ & 12,19 & 13,53 & $\mathbf{1 1 \%}$ \\
Sul / Sudoeste de Minas & 17,87 & 24,56 & $37 \%$ & 5,37 & 11,88 & $\mathbf{1 2 1 \%}$ \\
Triângulo Mineiro / Alto Paranaíba & 23,15 & 33,83 & $46 \%$ & 5,20 & 9,98 & $\mathbf{9 2 \%}$ \\
Vale do Mucuri & 9,02 & 12,17 & $35 \%$ & 0,07 & 3,88 & $\mathbf{5 6 5 7 \%}$ \\
Vale do Rio Doce & 10,92 & 15,15 & $39 \%$ & 2,69 & 5,99 & $\mathbf{1 2 3} \%$ \\
Zona da Mata & 14,59 & 19,51 & $34 \%$ & 2,06 & 8,16 & $\mathbf{2 9 6 \%}$ \\
\hline \hline
\end{tabular}

Fonte: Elaboração própria.

o modelo mais adequado para fins de análise.

O Modelo 1 foi estimado por MQO com a variável dependente defasada como variável explicativa. Observa-se que seu coeficiente estimado foi positivo e apresentou significância ao nível de $1 \%$. No entanto, pode-se rejeitar o modelo pelos testes de correlação serial de primeira e segunda ordem, que indicam a possivel omissão de variáveis de efeito persistente ao longo do tempo ou falta de controle para o efeito dinâmico dos suicídios.

Já o Modelo 2 foi estimado por efeitos fixos, de forma a controlar o efeito fixo de cada município ao longo do tempo. O coeficiente da variável dependente defasada foi positivo e apresentou significância ao nível de 1\%, comprovando o componente inercial das taxas de suicídio. No entanto, assim como no Modelo 1, os testes de especificação continuam apontando a presença de correlação serial. Por sua vez, o Modelo 3 (GMM-Difference) resultou em um coeficiente da variável dependente defasada estatisticamente significativo, no entanto com valor abaixo dos modelos estimados anteriormente. Além disso, persiste a presença de correlação serial.

Por fim, o Modelo 4 (GMM-System) não apresentou problemas em relação aos testes 
de correlação serial e de Sargan. Dessa forma, a estimação desse permite um ganho considerável de precisão em comparação aos demais modelo estimados. Portanto, esse é o modelo cujos resultados são analisados a seguir.

Tabela 4. Resultados econométricos

\begin{tabular}{|c|c|c|c|c|}
\hline Variável & $\begin{array}{l}\text { Modelo } 1 \\
\text { (MQO) }\end{array}$ & $\begin{array}{c}\text { Modelo } 2 \\
\text { (Efeitos } \\
\text { Fixos) }\end{array}$ & $\begin{array}{c}\text { Modelo } 3 \\
\text { (GMM- } \\
\text { Difference) }\end{array}$ & $\begin{array}{c}\text { Modelo } 4 \\
\text { (GMM- } \\
\text { System) }\end{array}$ \\
\hline \multirow{2}{*}{ CONSTANTE } & $0,9812956^{* * *}$ & $2,164284^{* * *}$ & $3,170604^{* * *}$ & $1,515614^{* * *}$ \\
\hline & $(0,0865531)$ & $(0,1652363)$ & $(0,5284113)$ & $(0,2289581)$ \\
\hline \multirow{2}{*}{$L N T X S U I C_{i, t-1}$} & $0,3857065^{* * *}$ & $0,2050406^{* * *}$ & $0,1951308^{* * *}$ & $0,3706779^{* * *}$ \\
\hline & (0.018408) & $(0,02057)$ & $(0,0497398)$ & $(0,0234102)$ \\
\hline \multirow{2}{*}{$T X E M P F O R M_{i, t}$} & $-0,0020532^{N S}$ & $-0,0194^{* * *}$ & $-0,1209261^{* * *}$ & ${ }^{*}-0,0541711^{* * *}$ \\
\hline & $(0,0017435)$ & $(0,0068)$ & $(0,0279509)$ & $(0,0085505)$ \\
\hline \multirow{2}{*}{$S A U ́ D E_{i t}$} & $0,0005916^{* * *}$ & $0,0008^{* * *}$ & $0,003103^{* *}$ & $0,0013975^{* *}$ \\
\hline & $(0,0001332)$ & $(0,00023)$ & $(0,0012962)$ & $(.0006766)$ \\
\hline \multirow{2}{*}{$A S S S O C I A L_{i, t}$} & $-0,0003049^{N S}$ & $-0,00058^{N S}$ & $-0,0087978^{*}$ & $-0,0006766^{*}$ \\
\hline & $(0,0005505)$ & $(0,0008)$ & $(0,004751)$ & $(0,001497)$ \\
\hline \multirow{2}{*}{$E D U C A C ̧ \tilde{A} O_{i, t}$} & $0,0008929^{* * *}$ & $0,0009^{* * *}$ & $0,0002373^{N S}$ & $0,0012188^{N S}$ \\
\hline & $(0,0001438)$ & $(0,0003)$ & $(0,0017028)$ & $(0.0010317)$ \\
\hline \multirow{2}{*}{ PIBPERCAPIT $A_{i, t}$} & $-0,000006^{* * *}$ & $0,000005^{N S}$ & $0,0000244^{* *}$ & $0,0000139^{N S}$ \\
\hline & $(0,000002)$ & $(0,000004)$ & $(0,0000111)$ & $(0,000009)$ \\
\hline \multirow{2}{*}{$D E N S D E M O G R_{i, t}$} & $-0,0001191^{* *}$ & $0,00119^{*}$ & $0,0006461^{N S}$ & $0,0010421^{*}$ \\
\hline & $(0,0000579)$ & $(0,0006)$ & $(0,0018255)$ & $(0,000544)$ \\
\hline \multirow{2}{*}{ EVELHECIMENTO ${ }_{i, t}$} & $0,0063557^{* * *}$ & $-0,01326^{* * *}$ & $0,0142529^{* * *}$ & $0,0076874^{*}$ \\
\hline & $(0,0016993)$ & $(0,00241)$ & $(0,004523)$ & $(0,0045466)$ \\
\hline \multirow{2}{*}{$T X M O R T A G R E S S_{i, t}$} & $0,0006852^{* *}$ & $0,00093^{* *}$ & $0,0015376^{*}$ & $0,0019589^{*}$ \\
\hline & $(0,0003003)$ & $(0,00041)$ & $(0,0008144)$ & $(0,0010874)$ \\
\hline Teste de Correlação Serial (1 $1^{\text {a }}$ Ordem) & 0,0000 & 0,0000 & 0,0000 & 0,0000 \\
\hline Teste de Correlação Serial (2 $2^{\text {a }}$ Ordem) & 0,0000 & 0,0000 & 0,0654 & 0,4096 \\
\hline Teste de Sargan & - & - & 0,3747 & 0,3922 \\
\hline
\end{tabular}

Nota: ${ }^{* *}$ significativo a $10 \%,{ }^{* *}$ significativo a $5 \% \mathrm{e}^{*}$ significativo a $1 \%$.

Fonte: Elaboração própria.

Com os resultados apresentados na Tabela 4, é possível perceber que a relação entre a Taxa de Suicídios e a Taxa de Emprego no Setor Formal é negativa e estatisticamente significativa no modelo analisado. Desse modo, vide Modelo 4, pode-se inferir que o aumento em $1 \%$ na taxa de emprego formal no município corresponde a uma redução de 5,41\% na Taxa de Suicídios da localidade. Esse resultado está em 
consonância com a proposição levantada no início deste trabalho.

Isso condiz com a explicação à luz da sociologia proposta por Durkheim (2000) sobre o suicídio anômico, em que o indivíduo, ao passar individual ou coletivamente por momentos de instabilidade, que vêm acompanhados de alterações inesperadas, acaba por se desconectar da realidade, deixando de deter identificação com o capital social, o que o leva a um estado infeliz e pode resultar no atentado contra a própria vida. Além disso, uma outra explicação proposta pelo autor considera que a facilidade de acesso aos recursos leva o indivíduo a ter menos limitações. Quando essa pessoa passa por uma mudança negativa repentina na vida financeira, que levando-a a viver sob mais limitações, ela pode desenvolver um quadro de ansiedade ou depressão, que pode resultar no suicídio.

Como aponta Oliveira et al. (2010), em função do lugar de destaque que o trabalho ocupa na vida das pessoas, sendo garantia de subsistência e forma de integração social, a falta deste ou até mesmo a ameaça de perda geram sofrimento psíquico, sentimento de menos-valia, angústia, insegurança, desânimo e desespero, podendo levar a quadros ansiosos e depressivos.

Uma importante constatação desse resultado é que, além da questão do emprego em si, o fato de o mesmo ser formal, com maior nível de estabilidade, diminui riscos de mudanças repentinas, as quais podem engatilhar situações extremas, como sugeriu Durkheim (2000). Conforme proposto por Kim e Cho (2017), a distinção entre emprego e desemprego não reflete todas as dimensões da instabilidade do mercado de trabalho que podem levar a situações de estresse. Os trabalhadores formais são assistidos por direitos os quais diminuem grandes riscos à sua sobrevivência, como seguro desemprego e licença maternidade. Além disso, Oliveira et al. (2010) destacam fatores como sobrecarga de trabalho e tempo insuficiente para lazer, comuns entre profissionais informais, também associados aos riscos de depressão, ansiedade e alcoolismo.

A variável SAUDE foi estatisticamente significativa e com sinal positivo no Modelo 4, o que a princípio pode não fazer sentido, mas, quando observada a relação endógena com a variável dependente, verifica-se coerência, tendo em vista que maiores taxas de suicídio podem ser motivo para políticas públicas que resultem em aumento nos gastos per capita com saúde. Já a variável ASSSOCIAL apresentou sinal negativo, o que vai ao encontro da expectativa inicial para esta variável.

A variável Gasto per capita com Educação foi adicionada com a expectativa de que fosse negativa e estatisticamente significativa, correspondendo com o estudo de Blakely et al. (2003). Entretanto, através da Tabela 4 , nota-se que a variável EDUCACÃO não é significativa no Modelo 4, que é aquele a ser analisado. Uma possível explicação se deve ao fato de que os Gastos na área da Educação têm diversos fins e aplicações, que não necessariamente resultam diretamente na prevenção dos suicídios.

Para a variável PIB per capita, essa se mostra não significativa no Modelo 4. Apesar de apresentar sinal distinto do esperado, esse resultado é explicável, pois, a partir de 
determinado nível de renda, as pessoas não necessariamente ficam menos propensas a apresentarem distúrbios mentais ou comportamentos suicidas. Isso ocorre, pois, como denotado por Durkheim (2000), o aumento da renda provoca uma falsa sensação de independência nas pessoas, que pode não ser sustentada permanentemente. Além disso, Hamermesh e Soss (1974) encontraram que a variável renda apresentou relação positiva com a taxa de suicídios para homens entre 20 e 34 anos. Segundo os autores, isso pode ser explicado pelo fato de que nessa faixa etária os indivíduos demandam mais educação e tendem a postergar o consumo de bens, serviços ou experiências desejáveis.

Como abordado anteriormente, a variável DENSDEMOGR apresentava ambiguidade em relação a seu impacto esperado sobre a Taxa de Suicídios, podendo, em alguns casos, ter interação positiva ou, em outros, relação negativa com a variável explicativa. Entretanto, através dos resultados encontrados percebeu-se que a DENSDEMOGR apresentou impacto levemente positivo, corroborando o que foi encontrado por Loureiro et al. (2013). Como apresentado na Tabela 4, infere-se que variações em $1 \%$ na densidade demográfica provocam aumento em aproximadamente $0,1 \%$ na Taxa de Suicídios, o que pode ser explicado pela maior propensão das pessoas em desenvolverem distúrbios mentais em locais com densidade populacional maior em relação àqueles menos populosos (Matheson et al., 2006).

Com relação à variável ENVELHECIMENTO, esperava-se uma relação positiva entre essa e a Taxa de Suicídios, o que de fato ocorreu e de forma estatisticamente significativa. No entanto, assim como no caso da variável DENSDEMOGR, o Envelhecimento também apresentou impacto moderado, de aproximadamente 0,77\%. Taxas de acometimento por transtorno depressivo costumam ser mais elevadas em idades avançadas, o que de acordo com Santos (2012) pode ser explicado pela redução das oportunidades e menor integração social inerentes ao envelhecimento. Além disso, segundo Machado e Santos (2015), as dificuldades de se suportar problemas de saúde ou pressões emocionais podem explicar os suicídios que ocorrem em indivíduos com idade acima dos 60 anos.

No caso da variável TXMORTAGRESS, também ocorreu resultado dentro do que era esperado, positivo, sendo que a referida taxa impacta a Taxa de Suicídios em aproximadamente $0,2 \%$. Isso possivelmente ocorre, pois a forte presença da violência pode gerar efeitos psicológicos graves relacionados, por exemplo, à perda de parentes e amigos, o que causa um rompimento emocional brusco (Costa et al., 2017; Dalbosco, 2006).

\section{Considerações Finais}

Diante da relevância do fenômeno do suicídio e das complicações sociais inerentes a esse problema, pretendeu-se com o presente estudo analisar em que medida o nível de emprego no setor formal impacta as taxas de suicídios no estado de Minas 
Gerais. Para tal, foi utilizado um modelo econométrico com dados em painel dinâmico estimado através do GMM-SYS para o período 2002-2017.

O resultado central da pesquisa confirmou a hipótese da existência de uma relação negativa entre as Taxas de Emprego no Setor Formal e as Taxas de Suicídios. De acordo com a literatura relativa ao tema, esse resultado pode refletir a pressão psicológica provocada pela falta de recursos derivada do desemprego, além da instabilidade derivada do emprego informal.

Com relação às demais variáveis de controle, somente as variáveis de Gastos per capita com educação e de PIB per capita não foram estatisticamente significativas a um nível de significância de pelo menos $10 \%$. Por sua vez, a única variável que apresentou relação inversa com a Taxa de Suicídios, além da Taxa de Emprego no Setor Formal, foi a de Gastos per capita com Assistência Social. Todas as demais variáveis de controle foram estatisticamente significativas. Nesse sentido, Gastos per capita com Saúde, Densidade Demográfica, Envelhecimento e Taxa de Mortes por Agressão apresentaram sinal positivo.

Diante dos resultados, percebe-se a complexidade do tema e a urgência para que medidas sejam tomadas com o intuito de reduzir as taxas de suicídios, que são alarmantes em alguns municípios. Em relação ao governo, é importante que esse se atente para a forma como são realizados os gastos em Saúde Pública, Assistência Social e Educação e que esses sejam alocados de forma mais eficiente, além de estabelecer um cenário macroeconômico propício à geração de empregos e à reinserção dos indivíduos no mercado de trabalho, com garantia de direitos e estabilidade trabalhista a esses, haja vista a importância psicológica que esses fatores possuem. Similarmente, as organizações voltadas para a prevenção do suicídio podem utilizar os resultados desta pesquisa para implementar possiveis medidas com foco nos indivíduos acometidos por depressão, ansiedade e outros distúrbios mentais, a fim de mitigar os efeitos desses problemas.

Por fim, é importante mencionar algumas limitações. Em primeiro lugar, outras variáveis que impactam a Taxa de Suicídios não foram utilizadas no trabalho, particularmente pela escassez de algumas informações para o período analisado. Além disso, não foi possível inserir variáveis dummies para denotar o efeito espacial das mesorregiões sobre a ocorrência de suicídios. Nesse caso, é importante ressaltar que a ocorrência de multicolinearidade prejudicou os resultados, impossibilitando a inserção das mesmas no modelo. Para pesquisas futuras, seria importante analisar a questão da duração do desemprego e seu impacto nas taxas de suicídios.

\section{Referências}

Arellano, M. e Bover, O. (1995). Another look at the instrumental variable Estimation of error components models. Journal of Econometrics, 68(1):29-51. 
Basta, M., Vgontzas, A., Kastanaki, A., Michalodimitrakis, M., Kanaki, K., Koutra, K., Anastaki, M., e Simos, P. (2018). Suicide rates in Crete, Greece during the economic crisis: the effect of age, gender, unemployment and mental health service provision. BMC Psychiatry, 18(1):39-52.

Bertolote, J. M. e Alexandra, F. (2002). A global perspective in the epidemiology of suicide. Suicidologi, 7(2):6-8.

Blakely, T. A., Collings, S. C. D., e Atkinson, J. (2003). Unemployment and suicide. Evidence for a causal association?. Journal of Epidemiology and Community Health, 57(8):594-600.

Blundell, R. W. e Bond, S. R. (1998). Initial conditions and moment restrictions in Dynamic panel data models. Journal of Econometrics, 87(1):115-143.

Chang, T. e Chen, W. Y. (2017). Revisiting the relationship between suicide and unemployment: evidence from linear and nonlinear cointegration. Economic Systems, 41(2):266-278.

Costa, A. G. e Ludemir, A. B. (2005). Transtornos mentais comuns e apoio social: estudo em comunidade rural da Zona da Mata de Pernambuco, Brasil. Cadernos de Saúde Pública, 21(1):73-79.

Costa, D. H., Njaine, K., e Schenker, M. (2017). Repercussões do homicídio em famílias das vítimas: Uma revisão da literatura. Ciência e Saúde Coletiva, 22(9):3087-3097.

Dalbosco, C. (2006). Ressonâncias da morte violenta de adolescentes e jovens: estudo teórico clínico de famílias em sofrimento. Chest, 25(1):1-14.

Durkheim, E. (2000). O suicídio: estudo de sociologia. São Paulo: Martins Fontes.

Fraga, W. S., Massuquetti, A., e Godoy, M. R. (2017). Determinantes Socioeconômicos do Suicídio no Brasil e no Rio Grande do Sul. Revista Econômica,32(2):1-20.

Gerdtham, U. G. e Ruhm, C. J. (2006). Deaths rise in good economic times: evidence from the OECD. Economics \& Human Biology, 4(3):298-316.

Godoi, E. F. (2018). Tese de Doutorado. Aspectos socioeconômicos e regionais das condições associadas a transtornos mentais e suicídio: uma análise da morbidade hospitalar e mortalidade. Monografia - Departamento de Ciência Econômicas da Universidade Federal de Minas Gerais. Belo Horizonte, p. 50.

Gonçalves, L. R. C. e Lourival, B. D. O. J. (2011). Determinantes espaciais e socioeconômicos do suicídio no Brasil: uma abordagem regional. Nova Economia,25(1):3672 .

Hamermesh, D. S. e Soss, N. M. (1974). An Economic Theory of Suicide. Journal of Political Economy, 82(1):83-98. 
Helbich, M., Blüml, V., Jong, T., Plener, P. L., Kwan, M. P., e Kapusta, N. D. (2017). Urban-rural inequalities in suicide mortality: A comparison of urbanicity indicators. International Journal of Health Geographics, 16(1): 1-12.

Iglesias-Garcia, C., Sáiz, P. A., Burón, P., Sánchez-Lasheras, F., Jiménez-Trviño, L., Fernandéz-Artamendi, S., Al-Halabi, S., Corcoran, P., Gárcia-Portilla, M. P., e Bobes, J. (2017). Suicide, unemployment, and economic recession in Spain. Revista de Psiquiatría y Salud Mental, 10(2):70-77.

Isometsä, E., Heikkinem, M., Henriksson, M., Marttunem, M., Aro, H., e Lönnqvist, J. (1997). Differences between urban and rural suicides. Acta Psychiatrica Scandinavica, 95(4):297-305.

Kaufman, J. A., Salas-Hernández, L. K., Komro, K. A., e Livingston, M. D. (2020). Effects of increased minimum wages by unemployment rate on suicide in the USA. Journal of Epidemiology and Community Health, 21(1):259-302.

Kim, C. e Cho, Y. (2017). Does unstable employment have an association with suicide rates among the young?. International Journal of Environmental Research and Public Health, 14(5):132-145.

Kposowa, A. J. (2001). Unemployment and suicide: a cohort analysis of social factors predicting suicide in the US National Longitudinal Mortality Study. Psychological Medicine, 31(1): 127-138.

Loureiro, A., Costa, C., Almendra, R., Freitas, A., e Santana, P. (2015). O contexto socioespacial como fator de risco de internação por doença mental nas áreas metropolitanas de Portugal. Cadernos de Saúde Pública, 31:S219-S231.

Loureiro, P. R. A., Moreira, T. B., e Sachsida, A. (2013). Tese de Doutorado. Os efeitos da mídia sobre o suicídio: uma análise empírica para os estados brasileiros. Texto para Discussão n.1851, IPEA.

Ludin, A., Lundberg, I., Allebeck, P., e Hemmingsson, T. (2012). Unemployment and suicide in the Stockholm population: A register-based study on 771,068 men and women. Public Health, 126(5):371-377.

Machado, D. B. e Santos, D. N. (2015). Suicídio no Brasil, de 2000 a 2012. Jornal Brasileiro de Psiquiatria, 64(1):45-54.

Massardi, W. O. e Abrantes, L. A. (2016). Dependência dos Municípios Mineiros em relação ao Fundo de Participação dos Municípios. Gestão \& Sociedade, 10(27):256281.

Matheson, F. I., Moineddin, R., Dunn, J. R., Creatore, M. I., Gozdyra, P., e Glazier, R. H. (2006). Urban neighborhoods, chronic stress, gender and depression. Social Science and Medicine, 63(10):2604-2616. 
Milner, A., Page, A., e Lamontagne, A. D. (2012). Long-Term Unemployment and suicide: A Systematic Review and Meta-Analysis. PLoS ONE, 8(1):659-667.

Morrell, S., Taylor, R., Quine, S., e Kerr, C. (1993). Suicide and Unemployment in Australia 1907-1990. Social Science \& Medicine, 36(6):749-756.

Nock, M. K., Borges, G., Bromet, E. J., Cha, C. B., Kessler, R. C., e Lee, S. (2008). Suicide and suicidal behavior. Epidemiologic Reviews, 30(1):133-154.

Oliveira, G. F., Carreiro, G. S. P., Ferreira Filha, M. O., Lazarte, R., e Vianna, R. P. T. (2010). Risco para depressão, ansiedade e alcoolismo entre trabalhadores informais. Revista Eletrônica de Enfermagem, 12(2):272-277.

Reeves, A., McKee, M., Gunnell, D., Chang, S. S., Basu, S., Barr, B., e Stuckler, D. (2015). Economic shocks, resilience, and male suicides in the Great Recession: Cross-national analysis of 20 EU countries. European Journal of Public Health, 25(3):404-409.

Santos, J. G. (2012). Sintomas depressivos e prejuízo funcional de idosos de um Centro-dia Geriátrico. Jornal Brasileiro de Psiquiatria, 6(1):102-106.

Santos, M. J. e Kassouf, A. L. (2008). Estudos Econômicos das Causas da Criminalidade no Brasil: Evidências e Controvérsias. Revista EconomiA, 9(2):343-372.

Stuckler, D., Basu, S., Suhrcke, M., Coutts, A., e McKee, M. (201 1). Effects of the 2008 recession on health: A first look at European data. The Lancet, 378(9): 124-125.

Tapia Granados, J. A. e Diez Roux, A. V. (2009). Life and death during the Great Depression. Proceedings of the National Academy of Sciences of the United States of America, 106(41):17290-17295.

Vieira, F. V., Verissimo, M. P., e Avellar, A. P. M. (2016). Indústria e Crescimento: Uma Análise de Painel para os Estados Brasileiros. Análise Econômica, 34(65):114-131.

World Health Organization (2002). World report on violence and health. Geneva.

World Health Organization (2014). Preventing suicide: a global imperative. Geneva.

World Health Organization (2017). Depression and Other Common Mental Disorders: Global Health Estimates. World Health Organization. Geneva.

World Health Organization (2019). Suicide in the world: Global Health Estimates. World Health Organization, Geneva, p. 1-33.

(G) Este artigo está licenciado com uma CC BY 4.0 license. 